\title{
Cognitive Radio Networks and Spectrum Sensing
}

\author{
A Review \\ https://doi.org/10.3991/ijoe.v16i13.18549 \\ Preet Talajiya, Aniket Gangurde, U Ragavendran, Hariharan Murali $\left.{ }^{\bowtie}\right)$ \\ SVKM's NMIMS MPSTME, Shirpur, India \\ anand13.hm@gmail.com
}

\begin{abstract}
The wireless spectrum demand is at a constant rise in contrast to its scarcity. Cognitive Radio Networks (CRN's) as a notion was first brought into the light to tackle this issue. A CRN is an intelligent network that dynamically changes its characteristics through the process of Spectrum Sensing and adapts to the convenience of the environment it is in. This article presents a basic overview of what CRN's are. The major contributions of this paper lie in a comparative study of CRN's and Spectrum Sensing in recent years as well as its main challenges and applications. This review shall help current and new researchers in the field to look for future outlooks and give them a basic run-through of CRN's and Spectrum Sensing and their characteristics.
\end{abstract}

Keywords - Spectrum Sensing, Cognitive Radio Network, Spectrum Allocation, Wideband Sensing

\section{$1 \quad$ Introduction}

It is quite evident that the demand for the radio spectrum for wireless communications has enlarged due to rapidly increasing mobile users and the invention of other communication devices. Data traffic generated by mobile users is going to increase every year by some amount and hence more spectrum resources are needed to compensate for this rise. Within the currently available radio spectrum, most of the bands are allocated for authorized administrations. But recent analysis and surveys say that up to $80 \%$ of the allocated bands are underutilized leading to a lot of Spectrum wastage [1]. This in turn has persuaded the Federal Communications Commission (FCC) to allow the utilization of authorized bands to unlicensed clients with the assistance of cognitive radio technology. A Cognitive Radio Network is the combination of a transmitter and receiver which naturally identifies accessible channels within the remote range and based on the result of detection it changes its transmission and gathering parameters so that more remote communications may run at the same time in a given range band [2]. According to the FCC of the United States Government, "A cognitive radio (CR) is a radio that can change its transmission parameters based on interaction with the environment in which it operates" [2]. 
The radio frequency spectrum has many users who exploit it to its farthest extent. These users are primarily categorized as Primary users (PUs) and secondary users (SUs). These are named terms that were given to users depending on their usage of the frequency spectrum [3]. A PU has the higher need or bequest rights on the utilization of a particular portion of the range whereas the SU has a lower need and so exploits the range in such a way that it does not impede the usage of the PU [4]. In this way, a PU is called a licensed user whereas a SU is called an unlicensed user. CR technology centers to use underutilized bands of a spectrum by allowing SUs to get the portion of the authorized spectrum which is not utilized by licensed clients at a certain time, in an opportunistic way [1]. Opportunistic spectrum access (OSA) implies that the SUs must identify unused portions of the licensed spectrum and use this licensed spectrum without interfering with PU communications. The CR networks are separated into two brackets: One is known as Single User and the other is known as Multi-User. Each family of Networks has its advantages and disadvantages. Also, based on the radio frequency band, we can have two types of CR networks namely single band or wideband (multiple bands).

The unused primary bands are known as spectrum holes [3] or white spaces. These white spaces can be accessed by secondary users. The process where the SUs continuously monitors the activity of the licensed users (PUs) to detect the spectrum holes is called spectrum sensing [3]. Based on their availability spectrum holes are of two types namely, temporal, and spatial. When a certain licensed band at a certain time is not being used for transmissions, a temporal spectrum hole is detected. As for a spatial spectrum hole, it occurs when the secondary user coverage area is not in the range of the primary user [3].

The radio spectrum has many frequencies which are widely used in Telecommunication. A frequency band, simply put, "is a range of frequencies between two limits". This means that a band can be defined as either a single frequency or several multifarious frequencies. Accordingly, there two types of bands: The single band is a band that works on a single frequency band and ceases to exist beyond this frequency. The other band is called a Multiband. The Multiband as its name suggests is a band that works on multiple frequency bands.

Radio Frequency bands of the Electromagnetic Spectrum are meant to be exploited by certain systems. These systems can broadly be divided into two classes by the number of operators working in the system: The Single-User and Multi-User systems. A Single User system is a system where only a single user makes use of the Spectrum available to it. It does not matter if there many stations or devices through which this user accesses the bands, as long it is the only User that exploits the bands. In a MultiUser System, there is always more than one user trying to benefit from the spectrum. As there are several operators/users, and each user has different or similar privileges over the frequency bands depending on its usage of the stations and devices available to them.

To make more effective use of the available radio frequency bands, a CR network with a fitting spectrum sensing strategy can be utilized. These CR networks are known to improve the rate of data transmission in future wireless communications networks. Dynamic spectrum access strategies utilizing cognitive radio technology could ease 
spectrum administration while expanding spectrum efficiency [2]. CRN covers multiple fields of research and it attracts a large amount of research work. There are many challenges faced when working with CRNs. These are implementation, intelligence, distribution, security, sensing algorithms, and adaptive hardware design.

Over the years various spectrum sensing techniques have been developed which are classified as follows:

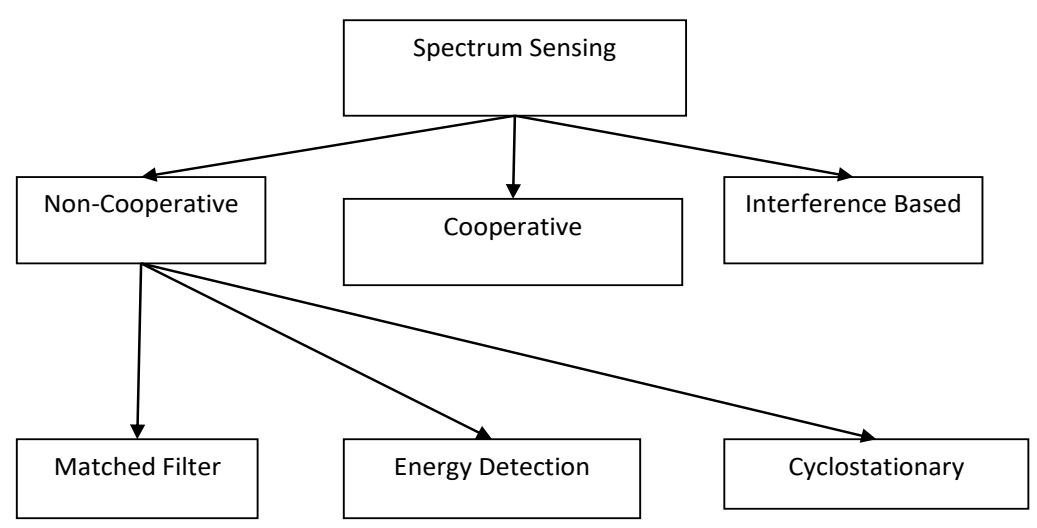

Fig. 1. Classification of Spectrum Sensing [6].

One of the topics we have focused on in our review is Cooperative Sensing which is one of the many ways Spectrum Sensing is performed. Cooperative Spectrum Sensing aids in improving the sensing ability of the CR Network, by taking in the results and sensing information of individual $\mathrm{CR}$ users and integrating them to improve the overall sensing capability. By cooperation, $\mathrm{CR}$ users can make combined decisions which are more accurate than the individual decisions. When it comes to aspects affecting Cooperative Sensing, there are 3 main aspects: 1 . How we approach cooperative sensing. 2. Models used to represent the sensing method and 3. Challenges faced when using this sensing method [5]. Cooperative Sensing follows 3 different approaches: Centralized, Distributed, and Relay-based approach. Models used to explain how cooperative sensing works are Game Theoretical model, Parallel Fusion, Machine Learning technique, Particle Swarm Optimization, and Random Matrix Theory [5]. Also, the challenges faced when working with Cooperative Sensing are Channel Uncertainty, Cooperation Overhead, Decision Fusion, Interference temperature measurement, Coalition Formation, and finally Mobility [5].

The main aim of our paper is to provide an insight regarding Cognitive Radio Networks and all the fundamentals associated with it. Radical terms are discussed in Section I. This review centers on distinctive types of spectrum sensing techniques used in Cognitive Radio Networks which are discussed in Section II. In Section III, Spectrum allocation and Spectrum assignment approaches are briefly discussed. Wideband spectrum sensing has been reviewed in Section IV. In Section V research challenges about Cognitive Radio Networks have been examined, also its scope and applications in the 
field of wireless communication have been explored in Section VI. Finally, Section VII concludes the paper.

\section{Spectrum Sensing}

\subsection{Spectrum sensing techniques}

Spectrum Sensing is one of the foremost critical features of a CRN. We know that CRs cannot blend into an environment, unless and until the CR senses and detects the system's characteristics. To help it with the sensing, CR's use a process called Spectrum Sensing. For the most part, spectrum sensing procedures are classified as transmitter detection, receiver detection, and interference temperature detection [11]. Transmitter detection is also called as Non-Co-operative spectrum sensing detection. In this technique, the CR continuously senses the waveform transmitted by the primary transmitter [11]. In the different spectrum sensing techniques that researchers have come up with down the years, in each technique, SUs apply hypothesis testing criteria to locate spectrum holes and keep track of PU transmission [3]. So far, researchers have come with two basic hypothesis testing criteria model: the Neyman-Pearson and the Bayes test [3]. To keep average costs low, the Bayes test is applied. The Neyman-Pearson hypothesis testing criteria are used to improve the detection probability (Pf). The transmitter detection techniques have their unique hypothesis model which is given as:

$$
\begin{gathered}
x(t)=n(t) \quad H(0) \\
x(t)=s(t)+n(t) \quad H(1)
\end{gathered}
$$

Consider a signal $\mathrm{x}(\mathrm{t})$ received by a Cognitive Radio Network $(\mathrm{CRN})$ and $\mathrm{n}(\mathrm{t})$ is the additive white Gaussian noise perceived by this signal. The hypothesis of the absence and presence of PU signals is denoted by $\mathrm{H} 0$ and $\mathrm{H} 1$, respectively. By studying the characteristics of $\mathrm{x}(\mathrm{t})$, Spectrum Sensing aims to determine between $\mathrm{H} 0$ and $\mathrm{H} 1$. The two probabilities, i.e. probability of detection (Pd) and the probability of false alarm (Pf) characterize the performance of detection. When $\mathrm{H} 1$ is true, the likelihood of the decision being $\mathrm{H} 1$ is denoted by probability $\mathrm{Pd}$, whereas if $\mathrm{H} 0$ is true, the likelihood of the decision being $\mathrm{H} 1$ is denoted by probability Pf [3]. Depending on Pd, the probability of miss-detection (Pm) can be obtained as follows:

$$
\mathrm{Pm}=1-\mathrm{Pd}
$$

This hypothesis model for transmitter detection is used to implement the following spectrum sensing techniques:

Matched filter detection: This makes use of an optimal linear filter, also known as a matched filter, to perform spectrum sensing through the detection of white spaces and holes in the spectrum of the PUs, which can then be used by the SUs. This technique is derived from a specific cross-correlating process between a known and an unknown signal where, based on the unknown signals SNR value, the known signal detects the presence of an unknown signal [11]. 
Energy detector: Energy Detection technique is one of the most rudimentary and essential approaches to spectrum sensing. This method uses an apparatus called an energy detector or radiometer to exploit white spaces or holes in the given frequency band. Energy Detection technique is also called a non-coherent detection technique [6].

Cyclo stationary feature detection: Generally, the modulated signals and the sine waves are coupled according to their statistical properties like mean, autocorrelation, etc. This process is called the cyclo stationary process. In situations where there is stochastic noise present randomly in the network, the cognitive radio through the aid of the cyclo stationary property, still manages to detect a modulated signal. With the aid of the Spectral Correlation function, the above features can be obtained [11]. Cyclo stationary feature detection is a time-consuming and complex technique, but it is tradedoff by giving better performance than energy detection [11].

Over the years, the Spectrum sensing techniques have been characterized into 3 major types [7]. Each of these techniques has been carefully assessed and their drawbacks have also been noted. The results from the frequent surveys on various factors in the different Spectrum Sensing methods have helped researchers to come up with the Energy Detection method as the most efficient spectrum sensing methods among the 3 [7].

We know that spectrum sensing is one of the vital parts for the formation of cognitive radio and it's no surprise that many researchers in recent times have tried to analyze and study its various aspects and the most prominent researches are done in the field of spectrum sensing. In a period of over 10 years, different spectrum sensing methods have been reviewed and researched. Various techniques such as Local spectrum sensing, hypothesis testing criteria, matched filter detector, energy detector, feature detector, etc. have been evaluated in the past few years.

\section{$2.2 \quad$ Co-operative spectrum sensing}

Cooperative Spectrum Sensing (CSS) is a facet of the Collaborative Spectrum Sensing method, where a group of CR networks interact with each other and distribute the information they garner for Spectrum Sensing [22]. This method is quite useful and considerably augments the accuracy of the CR's sensing, as each Network has more data about the spectrum vacancies and PU's. It is known that CSS particularly helps in circumstances where there is a lot of noise uncertainty. Mahmood A. Abdulsattar and Zahir A. Hussein in their paper "Energy Detection Technique for Spectrum Sensing in Cognitive Radio: A Survey" explained the working of CSS with a diagrammatic approach. The diagram below shows that CR1 and CR2 are in PU Tx's range [22]. The CR2 does not directly receive the PU Tx's signal because the house is blocking the transmitted signals from reaching CR2. Thus, CR2 resists Multipath and Shadow fading and does not detect all the PU Tx's transmitted Signal. The CR3 is the network which is not even in the of the PU Tx. Not only does the CR3 not know that the PU Tx is transmitting, but it also does not know about the PU Rx's existence and so tends to disrupt with the receiving aspect of the PU Rx. This situation is quite problematic, but with the help of CSS, which was first advocated by researchers Ghasemi and Sousain, can be mitigated [22]. As Cooperative Spectrum Sensing is all about each network sharing the sensed information with other networks, the best receiving network, CR1 can 
share its sensing results with the other Networks. As each network receives this sensed information, they can gradually improve their sensing of the PU Tx's signal. This is the fundamental aspect of Cooperative Spectrum Sensing.

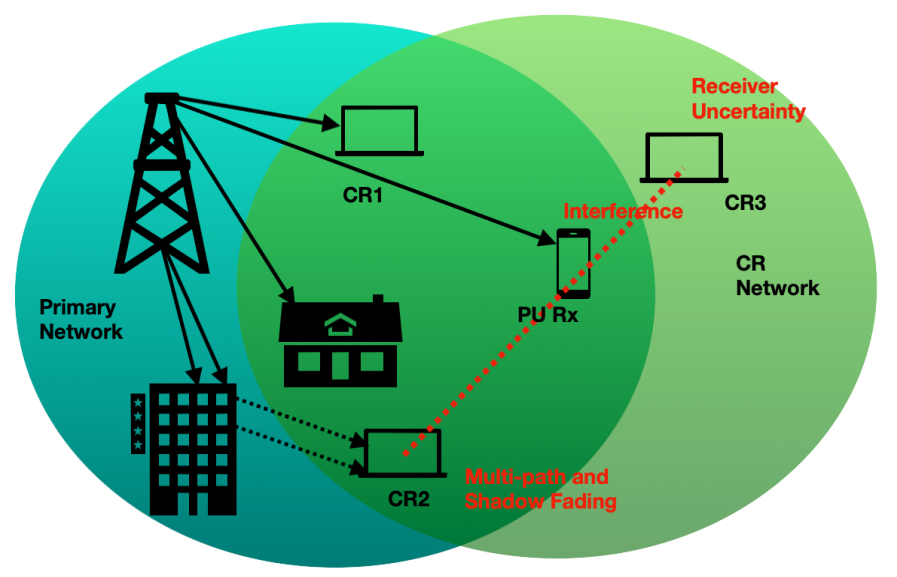

Fig. 2. A scenario where Co-operative Spectrum Sensing is applied [22].

There are broadly two approaches to co-operative spectrum sensing:

Centralized approach: Within the Centralized approach, there is a central CR called the fusion center (FC) [22]. The FC controls the method of cooperative spectrum sensing. The FC starts with selecting a frequency band to operate on and then it instructs all the cooperating CRs to individually perform spectrum sensing on the selected frequency band [6]. Once the cooperating CRs have finished sensing they send their sensing results back to the FC. Once the FC finishes analyzing the received results it then determines whether the PU is present or absent. This final decision is then propagated back to every CR in the vicinity.

Distributed approach: In the Distributed approach, unlike the centralized approach, there is no concept of a fusion center (FC) that makes the cooperative decision. [5]. Rather, in this approach, the CR users communicate with each other by exchanging sensing information and then come to a unified conclusion about whether the PU is present or not by iterations [5].

A superior and more advanced method of the Distributed CSS is the Distributed learning-based Multiband Multiuser Cooperative Sensing (M2CSS) [9]. This technique was proposed by researchers to improve the performance of detection and efficiency of energy use among different channels in the existing Distributed approach. The fundamental approach of M2CSS is to use the distributed approach scheme to select the foremost suitable SUs for channel detection. The M2CSS scheme allows the SUs in the band sense multiple channels at a time and this technique comprises of two stages [9]:

1. Selection of a Leader for each channel

2. Selection of the cooperative SUs to sense the selected channels. 
The leaders for each channel are selected such that, there is only one SU that can be the leader for a channel and that the selected leader can communicate effectively with the other SUs in its vicinity. In the next stage, cooperative SUs are selected for each channel so that CSS can be performed optimally [9]. Many researchers have suggested that the M2CSS technique not only improves the detection performance of the entire network but also ignores the choice of repetitive cooperative SUs. It is also that, the energy provided is fairly consumed among the different channels because of the implementation of M2CSS [9].

\section{Spectrum Allocation}

\subsection{Spectrum assignment}

When concerned with interference, spectrum assignment (SA) in CRNs is very distinctive from spectrum assignment in conventional wireless networks. Not only PUs may get affected by a significant amount of interference because of an SU but other SUs might also be affected due to these phenomena. To address this issue, an efficient spectrum assignment method widely known as spectrum allocation for CRN has been considered. Channel assignment in CRNs is characterized as a solution for determining an ideal plotting between the free licensed channels i.e. PUs and the SUs such that the performance is optimal. Compared to channel allocation in conventional wireless networks, this approach is more convoluted. It also deals with numerous design issues such as fault tolerance, interference, stability, throughput, and connectivity [7], [10]. Spectrum assignment can be explained as the plotting of the spectrum or the channel to the radio interfaces, to accomplish the ideal utilization of channel and at the same time maintaining the slightest channel interference [10].

Channel assignment is generally classified into three categories:

Fixed or Static: In the fixed channel assignment, the assignment configuration of the channels is almost constant or fixed [10].

Dynamic: In a dynamically assigned channel, the mapping of the channel is constantly updated with the changing network conditions [10].

Hybrid: A hybrid scheme adapts the behavior of both the static and the dynamic. Some portions of the channel are kept fixed while the rest of the portions adapt dynamic spectrum assignment [10].

\subsection{Spectrum assignment approaches}

The spectrum assignment schemes can moreover be categorized based on the following implementation methods:

Centralized approach: In a centralized spectrum assignment [7], [10], a central server is the one that performs all the activities and actions. It takes decisions and accordingly assigns the underutilized channels to SUs either periodically or on-demand. This central server is also called a spectrum server or a spectrum broker or a central base station [10]. This garners spectrum and radio information and knowledge from all 
SUs in the network. This approach of spectrum allocation has its many advantages such as it is quite easy to optimize the throughput of the entire network and also minimize the effect of interference [10]. The configuration of the centralized channel assignment approach is quite elementary and easy to understand, but the drawback of this approach is that it induces signal overhead in the network, which happens due to the exchanging of information continuously happening between the SUs and the central base node [10]. It is not robust to spectrum server failures during circumstances if the server crashes or there is a power failure.

Distributed approach: When it comes to distributed cognitive spectrum assignment, there is no such thing as a central entity like in the centralized spectrum assignment approach. Here the decisions are taken by the nodes themselves or they are taken by cooperating with their neighbors. Each node calculates the appropriate channels for all of the links by using only the local information available to them [10]. Distributed SA is ordinarily more adaptable and faster than a centralized SA approach because it can rapidly adjust to conceivable changes or network blackouts. After all, only SUs in the affected area will have to make changes and exchange information. Unlike in a distributed approach, in a centralized approach, only neighbor nodes exchange their information and thus it procures a lower signaling overload in the network [10]. The drawback of this distributed approach is that fairness is achieved only for a group of neighboring SUs. Distributed has its fair share of disadvantages. One such issue is that, because the final decision is based on the exchanging of information between the SUs [10] in the network, clients with malicious intent can share wrong information so that it can abuse the band available to them for their benefit. Distributed cognitive spectrum assignments can usually take adequate decisions in cases of low traffic load [10].

Cluster-based approach: We know there are two widely known methods of Cognitive Spectrum allocation which have their fair share of advantages and disadvantages. Thus, over the years, many researchers have tried to come up with an approach that neglects the disadvantages of both distributed and centralized and rather coalesces [10] their advantages to create a hybrid solution. This approach is named a Cluster-Based approach. In this approach, the entire network is divided into small divisions called clusters. Each of these clusters is assigned a personal router which is also called a cluster head. Here each of the routers independently and continuously senses information from the SUs and finally adds all the sensed values to come up with a vector called SA vector [10]. The heads exchange these information vectors and decide which channel to utilize and then relays the final decision taken to every node in the cluster. ClusterBased Approach has numerous advantages to it. One of them is that this approach is quite sturdy when it comes to router failures. This is because there is no one central server that makes the final decision, but a bunch of them, so even if one of them stops working the entire network does not falter. Another vital aspect of this approach is the fact that the bandwidth utilization is better than the other approaches. This is because the users are distributed into clusters and thus distributing the total load into multiple channels [10]. 


\subsection{Spectrum assignment techniques}

Commonly used spectrum assignment techniques that have been developed over the years by many eminent researchers are game theory, linear programming, non-linear programming, heuristics, network-based graph, genetic algorithms, evolutionary algorithms, and soft computing [7], [10]. These are advanced and convoluted channel assignment techniques that have been adopted in CRNs.

\section{$4 \quad$ Wide Band Spectrum Sensing}

Most of the efficient Spectrum Sensing techniques that we know of are only meant to be applied on a narrow band of the frequency spectrum. Initially, in narrowband sensing, a single band of the frequency spectrum is selected out and Spectrum Sensing is applied to it. This can be quite an arduous process in the long term. Hence, to tackle this issue, another class of spectrum sensing has been come up by researchers. This class is called Wideband Sensing. As its name suggests, wideband sensing in comparison to narrowband sensing allows us to analyze a larger band of the Radio Spectrum at a single time. According to Luca De Vito, in his paper "Methods and technologies for wideband spectrum sensing", "the problem of Wideband Spectrum Sensing consists of observing a wideband $\Delta \mathrm{f}$ and identifying the portions of such band which is occupied by a signal and those which are free" [23]. This can be quite helpful in the long run as De Vito mentions it to be a 'decisive factor' in its implementation of Spectrum Sensing equipment. But De Vito, in his paper, also mentions how difficult it is to come up with an architecture for applying wideband Sensing as it requires an enormously quick ADC and a DSP hardware [23]. Many researchers have come up with several wideband sensing methods of which, the usage of a bank of tunable frequencies is the most popular prospect. These methods have their drawbacks which De Vito explains in great detail in his paper.

\section{Research Challenges}

The various challenges that many researchers have faced throughout the years when delving into the practical realization of cognitive radio networks are as follows: 


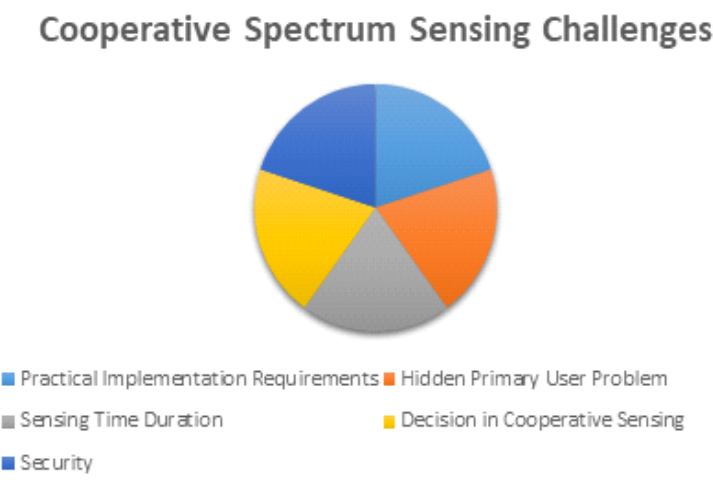

Fig. 3. Cooperative Sensing Challenges [1]

\subsection{Requirements when practically implemented}

CRN technology has numerous applications. But these applications require few essential factors so that they can optimally be implemented and used. Some of these factors include a rapid and quick signal processor, a high sampling rate, and an ADC with high resolution. Aside from that, the receiver should be able to perform fast processing. While keeping all the factors into consideration, we should make sure we achieve high accuracy as well as maintain an optimal receiver design [1].

\subsection{Hidden PU}

There may be situations when the SU fails to detect the PU's presence. This can lead to numerous issues and is one of the biggest challenges faced when implementing CRN technology. According to previous research, this issue occurs when various multipath and shadowing effects impede when looking for PU transmissions [1].

\subsection{Sensing time duration}

The fundamental goal of traditional spectrum sensing schemes is to keep track of the PUs activities. There may be times when an SU is exploiting the band of a licensed user opportunistically. As the PUs have full approbation over the band, they can come back online and use the band again. At this time, the SU needs to perceive this activity of the $\mathrm{PU}$ as soon as possible and vacate the band that they are exploiting, to avoid interference between the two users. The execution of conventional spectrum sensing schemes is restricted by this specified condition which then leads to the generation of a real problem which is for the real-time plan of CR systems [1]. 


\subsection{Spectrum sensing decisioning}

In CSS, a fusion center (FC) collects information from all the SU's in the network to elicit some useful information about the PU's presence through a process called Decision Fusion [1]. This task of collecting data from each SU is quite demanding because the FC has no clue which of the SUs in the vicinity are in cooperation with it or not [1].

\subsection{Security}

Over the years, in the CR technology field, researchers have advanced with the assumption that all users are honest and genuine and do not hold a threat towards the network. But that has never been the case and it is quite evident that CR networks similar to existing wireless networks are vulnerable to security threats. One of these threats is a malicious user that hides its original identity and takes the form of an existing PU user in the network. This user then further proceeds with its agenda of deluding the spectrum sensing technique in use. This behavior shown by this threatening user is called the Primary User Emulation Attack [1]. Another security threat that a CRN can face is reporting of false spectrum sensing information from a non-cooperative $\mathrm{SU}$ in the network. This can in turn lead to a wrong final decision and cause huge problems across the CRN. Because of the constant threats that wireless networks are prone to, security is one such aspect that is constantly receiving growing interest in CR networks. In light of these threats, researchers have come up with numerous security procedures such as cryptography and authentication [1]. But it is still not enough, because it is quite evident that these security threats are becoming smarter and difficult to deal with. Thus, to improve the security of CRNs and make it robust to malicious threats, either the current Spectrum sensing schemes need to be improved or better spectrum sensing schemes need to be developed.

\subsection{Practical hardware limitation}

We know that most of what we understand and comprehend about cognitive networks as a whole is mainly from a theoretical belief. But there have been some works where the theoretical aspects have been practically implemented for CR Networks. For the further development of Spectrum sensing plans in CRNs, these projects and works have been dealt with as the basis for the hardware and software for these systems [1]. In light of the ever-increasing potential of technology, more such works need to be done so that, CRNs can become more prevalent in the current scenario.

\section{Social Impact}

CRN technology is an anticipated technology in the telecommunication industry, and it has its fair share of applications which can be monumental help in today's scenario. Some of these applications that will use CR technology at its best are: 


\subsection{TV whitespaces}

Just as how frequency bands used for wireless communication have white spaces or spectrum holes that can be exploited using spectrum sensing, TV bands also have TV white spaces that go unused and thus can be exploited. Over the years, many administrative agencies across the globe such as the FCC [3], Ofcom, have tried to pursue the usage of these TV white spaces for wireless communication. And successfully in 2010, the FCC finally managed to get their hands on these white spaces, due to some monumental research in the field. In turn, they released a set of rules on how to exploit these TV whitespaces mainly using CRN technology [3].

\subsection{Healthcare system}

Nowadays, in the health care system more commonly in the field of telemedicine [21], wearable body sensors are more often being used. These are used to obtain intricate and analytical data of the patient's body. The coalescing of CR's with these body wearable sensors can be used to mitigate many of its current drawbacks in obtaining medical data which is not only critical but also delayed and error sensitive [21]. The inclusion of CR's will improve its global operability by solving problems of bandwidth and jamming and inevitably improve reliability.

\subsection{Cellular networks}

In recent years there has been a massive emergence of applications where CR technology helps. One such application is the concept of femtocells. The standard IEEE 802.16m and companies like PicoChip [3] came with this concept to tackle the indoor coverage problem and address the recurring issue of increasing traffic in wireless communications [3]. Typically, these small cells function as base stations but with the help of CR technology their usage can be enhanced to avoid interference and locate spectrum bands to maintain the desirable coverage.

\subsection{Bandwidth intensive applications}

The current networking industry has managed to offer so many bandwidth-intensive applications such as video conferencing, Online backing, YouTube, etc [21]. Because they take up a lot of bandwidth, they are extremely challenging to cope with for most around the world. Many other applications such as surveillance and tracking are also quite a bandwidth-hungry. Because in CR technology [21], the SU's can exploit multiple channels opportunistically, and whenever necessary, they can handle these bandwidth-intensive applications with ease.

\subsection{Emergency networks}

Extreme situations and emergencies are quite common in today's society and it is quite important to have reliable communications during such circumstances. E.g., 
during natural disasters its quite imminent to have good communication between the survivors and the responders. The current available wireless communication system is not yet capable to match these requirements. But with the help of CR technology, emergency network transmission can be enhanced so as minimize the damage in emergencies. The FCC has designated the frequency bands $698-806 \mathrm{MHz}$ for emergency usage and with the further help of CR technology, there will be more bands available during times of crisis.

\subsection{Military and public security usage}

CRN's have a vast application in the Military and Public Security Sector [21]. We know traditional wireless sensor networks are used for many purposes such as command control, battlefield surveillance, intelligent assistant, targeting, gathering information about the amount of battle damage, etc. But sometimes, it is quite imminent that the adversary might send some jamming signals to disturb normal radio communication channels. This can be a huge strategic deterrent. But this can also be overturned with the help of CRN's in use as CRN's can pass on frequencies over a wide range and that CRN's can use more than 1 frequency band, thereby neglecting the frequency band in which the jamming signal resides.

\section{Conclusion}

In the current scenario, the frequency bands that are available to us are not being used to their optimum extent and are quite inefficient. Thus, CR technology has been studied to tackle these inefficiencies and augment spectrum utilization efficiency. The Spectrum Sensing methods discussed in this paper help the SU's to detect the actions of the PU's. As single SU's have their fair share of drawbacks, we have also studied how CSS is a vital technique that collectively helps in Spectrum Hole Detection. We have also addressed how efficient spectrum assignment or spectrum allocation is used to plot a map b/w the SU's and PU's. Its different categories have also been briefed and its basic approaches as well. We have also noted some parts of Wideband Spectrum Sensing. Lastly, common challenges that spectrum sensing in general faces and the most prevailing applications that have come from CRN have also been cased in the paper.

\section{Acknowledgements} tion.

The authors of the paper have contributed equally and should get an equal contribu- 


\section{References}

[1] Mani Shekhar Gupta and Kishan Kumar, "Progression on spectrum sensing for cognitive radio networks: A survey, classification, challenges, and future research issues", Journal of Network and Computer Applications, vol. 143, pp. 47-76, October 2019. https://doi.org/10. 1016/j.jnca.2019.06.005

[2] Md Jakir Hossain, "Simulation of a cognitive radio system by using MATLAB".

[3] Xiangwe Zhou, Uzoma Onunkwo, Geoffrey Ye Li, (2012), "10 Years of research in spectrum sensing and sharing in cognitive radio", EURASIP Journal on Wireless Communications and Networking, Article number 28, January 2012. https://doi.org/10.1186/1687-14992012-28

[4] S. Shanmugavel, M. A. Bhagyaveni, and R. Kalidoss, "Cognitive Radio, An Enabler for Internet of Things", River Publishers Series in Communications.

[5] M. Sharma, P. Chauhan, and N. Sarma, "A brief review of cooperative spectrum sensing: Issues and challenges," 2016 International Conference on Accessibility to Digital World (ICADW), Guwahati, pp. 113-116, December 2016. https://doi.org/10.1109/icadw.2016. 7942523

[6] V. Amrutha and K. V. Karthikeyan, "Spectrum sensing methodologies in cognitive radio networks: A survey," 2017 International Conference on Innovations in Electrical, Electronics, Instrumentation and Media Technology (ICEEIMT), Coimbatore, 2017, pp. 306-310, February 2017. https://doi.org/10.1109/icieeimt.2017.8116855

[7] A. Mandal and S. Chatterjee, "A comprehensive study on spectrum sensing and resource allocation for cognitive cellular network," 2017 Devices for Integrated Circuit (DevIC), Kalyani, pp. 100-102, March 2017. https://doi.org/10.1109/devic.2017.8073915

[8] T. Xiong, Y. Yao, Y. Ren, and Z. Li, "Multiband Spectrum Sensing in Cognitive Radio Networks with Secondary User Hardware Limitation: Random and Adaptive Spectrum Sensing Strategies," in IEEE Transactions on Wireless Communications, vol. 17, no. 5, pp. 3018-3029, May 2018. https://doi.org/10.1109/twc.2018.2805729

[9] A. Gharib, W. Ejaz, and M. Ibnkahla, "Distributed Learning-Based Multi-Band Multi-User Cooperative Sensing in Cognitive Radio Networks," 2018 IEEE Global Communications Conference (GLOBECOM), Abu Dhabi, United Arab Emirates, 2018, pp. 1-6. https://doi. org/10.1109/glocom.2018.8648118

[10] Wangjam Singh and Marchang Ningrinla, "A Review on Spectrum Allocation in Cognitive Radio Network", International Journal of Communication Networks and Distributed Systems, pp. 172-193, March 2018.

[11] S. Ziafat, W. Ejaz, and H. Jamal, "Spectrum sensing techniques for cognitive radio networks: Performance analysis", 2011 IEEE MTT-S International Microwave Workshop Series on Intelligent Radio for Future Personal Terminals, Daejeon, 2011, pp. 1-4. https://doi.org/10. 1109/imws2.2011.6027191

[12] T. E. Bogale, L. Vandendorpe, and L. B. Le, "Wide-Band Sensing and Optimization for Cognitive Radio Networks with Noise Variance Uncertainty," in IEEE Transactions on Communications, vol. 63, no. 4, pp. 1091-1105, April 2015. https://doi.org/10.1109/tcomm. 2015.2394390

[13] Z. Quan, S. Cui, A. H. Sayed, and H. V. Poor, "Wideband Spectrum Sensing in Cognitive Radio Networks,” 2008 IEEE International Conference on Communications, Beijing, 2008, pp. 901-906. https://doi.org/10.1109/icc.2008.177

[14] Chintha Tellambura, \& Sachitha Kusaladharma,(2017), "An Overview of Cognitive Radio Networks", J. Webster (ed.), Wiley Encyclopaedia of Electrical and Electronics Engineering.Copyright(C2017 John Wiley \& Sons, Inc. https://doi.org/10.1002/047134608x.w8355

[15] Z. Jin, K. Yao, B. Lee, J. Cho, and L. Zhang, "Channel Status Learning for Cooperative Spectrum Sensing in Energy-Restricted Cognitive Radio Networks", in IEEE Access, vol. 7, pp. 64946-64954, 2019. https://doi.org/10.1109/access.2019.2916065 
[16] A. Mukherjee and A. Lee Swindlehurst, "Prescient beamforming in multi-user interweave cognitive radio networks", 2011 4th IEEE International Workshop on Computational Advances in Multi-Sensor Adaptive Processing (CAMSAP), San Juan, 2011, pp. 253-256. https://doi.org/10.1109/camsap.2011.6135996

[17] Y. An, Y. Xiao and G. Qu, "Multi-band spectrum auction framework based on location information in cognitive radio networks", in Journal of Systems Engineering and Electronics, vol. 23, no. 5, pp. 671-678, Oct. 2012. https://doi.org/10.1109/jsee.2012.00083

[18] B. Wang and K. J. R. Liu, “Advances in cognitive radio networks: A survey”, in IEEE Journal of Selected Topics in Signal Processing, vol. 5, no. 1, pp. 5-23, Feb. 2011.

[19] Van Tam Nguyen, F. Villain, and Y. Le Guillou, "Cognitive radio systems: Overview and challenges", 2011 3rd International Conference on Awareness Science and Technology (iCAST), Dalian, 2011, pp. 497-502. https://doi.org/10.1109/icawst.2011.6163179

[20] Y. Peng, J. Hao, Z. Yu, s. Yan and y. Wang, "A cognitive radio network spectrum balanced allocation algorithm combined spectrum sensing and priority", International Conference on Cyberspace Technology (CCT 2014), Beijing, 2014, pp. 1-4. https://doi.org/10.1049/cp. 2014.1294

[21] G. Joshi, S. Nam, and S. Kim, "Cognitive Radio Wireless Sensor Networks: Applications, Challenges and Research Trends", Sensors, vol. 13, no. 9, pp. 11196-11228, Aug. 2013. https://doi.org/10.3390/s130911196

[22] Mahmood A. Abdulsattar, Zahir A.Hussein, "Energy Detection Technique for Spectrum Sensing in Cognitive Radio: A Survey", International Journal of Computer Networks \& Communications (IJCNC) Vol.4, No.5, September 2012. https://doi.org/10.5121/ijenc. 2012.4514

[23] Luca De Vito, "Methods and technologies for wideband spectrum sensing", Journal of the International Measurement Confederation (IMEKO), November 2013.

\section{Authors}

Hariharan Murali is pursuing his bachelor's in technology Degree in Electronics and Telecommunication from SVKM's NMIMS Mukesh Patel School of Technology, Management, and Engineering, Shirpur. Telecommunication and its future are something he holds in high regard for. His keen interests in research are in computer networks and wireless communication.

Preet Talajiya was born in Mumbai, India. He is pursuing a B. Tech degree in Electronics and Telecommunication Engineering from the SVKM's NMIMS Mukesh Patel School of Technology, Management, and Engineering, Shirpur. His main research interests are in the field of computer networks, wireless communication, microcontrollers, and microprocessors. Email ID-talajiyapreet@gmail.com

Aniket Gangurde is pursuing his Bachelor of Technology degree majors in Electronics and Telecommunication Engineering from SVKM's NMIMS Mukesh Patel School of Technology Management and Engineering, Shirpur. His main research field of interest lies in Wireless Communication, Ad-HOC networks, and Embedded Systems. Email ID-aniket15gangurde@gmail.com

Article submitted 2020-09-14. Resubmitted 2020-10-23. Final acceptance 2020-10-28. Final version published as submitted by the authors. 